\title{
Neuromodulación sacra en el tratamiento de la incontinencia fecal*
}

\author{
Drs. CLAUDIO WAINSTEIN G. ${ }^{1}$, ANDRÉS LARACH K. ${ }^{1}$, FRANCISCO LÓPEZ K. ${ }^{1}$, \\ JORGE LARACH S. ${ }^{1}$, PEDRO MEDINA T. ${ }^{1}$, CRISTÓBAL SUAZO L. ${ }^{2}$, CLAUDIO HEINE T. ${ }^{1}$ \\ 1 Unidad de Coloproctología, Clínica las Condes. \\ 2 Departamento de Cirugía, Hospital Barros Luco Trudeau, Facultad de Medicina Universidad de Chile. \\ Santiago, Chile
}

\section{Sacral nerve stimulation in the treatment of faecal incontinence}

\section{Introducción}

La incontinencia anal o fecal se define como la incapacidad de postergar la defecación en forma voluntaria, produciéndose una pérdida recurrente e involuntaria de las heces (líquidas y/o sólidas) y/o gases a través del canal anal. Incluso en los casos leves, donde sólo se produce pérdida ocasional de gases y/o escurrimiento menor ("soiling"), esta patología se asocia en mayor o menor grado con compromiso en la calidad de vida de los pacientes en todos los ámbitos de su quehacer ${ }^{1}$.

La incontinencia anal es una patología multicausal que está presente en un porcentaje cada vez más importante de nuestra sociedad, debido al sostenido envejecimiento de la población. En series internacionales y nacionales, se estima que el 2 al $7 \%$ de los mayores de 18 años pueden presentar incontinencia anal, cifra que se eleva hasta el 35\% en pacientes de la tercera edad, especialmente en casas de reposo y hogares de ancianos ${ }^{2}$. Los principales factores de riesgo son: edad, menopausia, cirugías previas, parto traumático, fórceps, enfermedades del aparato conectivo, alteraciones neurológicas degenerativas y obesidad ${ }^{3,4}$.

El estudio de esta patología incluye una comple- ta anamnesis para evaluar factores de riesgo y antecedentes mórbidos, evaluación del tipo de tránsito intestinal y consistencia de las heces, alimentación y medicamentos de uso habitual.

Dentro del algoritmo de estudio, se debe evaluar la integridad anatómica y funcional del aparato esfinteriano, lo cual se logra con endosonografía anorrectal y manometría anal. Asimismo se puede complementar con estudio neurológico y electromiografía anal y tiempo de latencia de nervios pudendos. Debe descartarse además patologías como enfermedades inflamatorias intestinales, presencia de diarreas crónicas y pólipos o tumores secretores de moco realizando una colonoscopia completa.

En los pacientes en que se demuestra una alteración anatómica (sección) del esfínter externo en la endosonografia anal, la esfinteroplastía (plastia muscular) anal es hoy en día una de las mejores alternativas de tratamiento. Sin embargo, esta cirugía presenta un porcentaje de recurrencia, no menor al $40 \%$ a largo plazo ${ }^{5}$. Los pacientes que no presentan daño esfinteriano, o quienes han sido reparados sin éxito, pueden verse beneficiados con la implantación de un esfínter artificial, uso de neuromodulación sacra, o en casos muy adversos, con una ostomia definitiva. Lamentablemente la im-

\footnotetext{
*Recibido el 24 de Marzo de 2009 y aceptado para publicación el 21 de Abril de 2009.

Correspondencia: Dr. Claudio Wainstein G.

Lo Fontecilla 441 Edificio C3. Santiago, Chile. Fax: 6104776

E-mail: cwainstein@clc.cl
} 
plantación del esfínter artificial se asocia a un alto costo y a una tasa de complicaciones y/o retiro por infección muy elevadas ${ }^{6}$.

Dentro de las terapias actuales más novedosas se encuentra la neuromodulación de plexo sacro (NMS). Esta técnica fue inicialmente descrita por Tanagho y Schmidt, en $1981^{7,8}$ para tratar disfunciones urinarias. En estos pacientes se observó que, mediante estímulos eléctricos de los plexos sacros, se producía una mejoría en sus hábitos defecatorios y en la continencia anal. Posteriormente, es Matzel quien, en 1995, la utiliza por primera vez en incontinencia anal pura ${ }^{9}$. Desde entonces se han publicado diversas series con muy buenos resultados.

Basados en la experiencia internacional, en Noviembre de 2008, la Unidad de Coloproctología de Clínica Las Condes (UCPCLC) dio inicio a un protocolo prospectivo de tratamiento de incontinencia fecal con terapia de NMS. Este protocolo fue aprobado por el comité de ética de nuestra institución antes de su implementación clínica.

El objetivo de esta publicación es comunicar un recurso técnico quirúrgico distinto, junto al resultado a corto plazo del primer caso de incontinencia fecal tratado con neuromodulación sacra con la implantación de un marcapaso definitivo en nuestro país.

\section{Caso clínico}

Paciente de 51 años, de sexo femenino, multípara de cuatro, dos partos por cesárea y uno de ellos con fórceps, operada por incontinencia urinaria mixta en dos oportunidades. Consulta en UCPCLC por reaparición de incontinencia mixta de orina, incontinencia anal a gases y deposiciones sólidas, sensación de vaciamiento rectal incompleto y bulto vaginal. En mayo de 2005, se confirma una interrupción completa del esfínter anal externo, por lo que es sometida a una plastía muscular de esfínter anal externo con técnica de traslape y colpoperineoplastía posterior con malla compuesta (Ultrapro ${ }^{\circledR}$ ) en octubre del mismo año. La paciente evoluciona satisfactoriamente en su postoperatorio. En su evaluación kinesiológica post operatoria en abril de 2008, se constató escape de deposiciones, especialmente post evacuación y urgencia defecatoria, con persistencia de hipotonicidad esfinteriana, a lo que se agrega en esta oportunidad, disminución de la fuerza de contracción del esfínter anal externo y puborrectal. Los registros electromiográficos se corresponden con los hallazgos clínicos. A la manometría se observa una sensibilidad rectal aumentada, con una capacidad no mayor a $130 \mathrm{ml}$. Dados estos hallazgos se inicia entrenamiento muscular del piso pélvico apoyado por biofeedback. Posteriormente se efectuó una reevaluación clínica, destacando menor incontinencia a gases, sensación de escurrimiento anal una a dos veces a la semana, asociándose a esto incontinencia de orina de esfuerzo y urgencia miccional. Al examen físico destaca ano hipotónico, contracción voluntaria no satisfactoria y tacto vaginal normal. La endosonografía de control reveló continuidad del esfínter externo en sus tercios inferior y medio, con un índice de incontinencia de Wexner igual a 20. Dada su evolución y hallazgos clínicos de incontinencia recidivada con integridad de esfínter anal externo se plantea posibilidad de uso de terapia de neuromodulación sacra (NMS).

En diciembre de 2008 se realiza implante de electrodo estimulador sacro $\mathrm{S}_{3}$ derecho sin complicaciones. Desde la mañana siguiente a la cirugía, la paciente refiere desaparición de urgencia miccional. Controlada a los 3 y 7 días post implante refiere desaparición de incontinencia y urgencia urinaria y mayor control esfinteriano anal, con disminución de más de un $50 \%$ de episodios de incontinencia fecal. Se decide la colocación de marcapaso definitivo, procedimiento que se lleva a cabo sin complicaciones. Con tres meses de seguimiento post implante, la paciente evoluciona satisfactoriamente, con buena continencia anal, presentando un índice de incontinencia de Wexner igual a 3.

\section{Técnica quirúrgica}

La neuromodulación sacra (NMS) consta de 2 etapas:

a) Fase temporal o de prueba: Se localiza una de las raíces sacras $\mathrm{S}_{2}, \mathrm{~S}_{3}$ o $\mathrm{S}_{4}$ y se estimulan durante un período variable de 5 días a 2 semanas mediante un estimulador externo.

b) Fase crónica, definitiva o terapéutica: Colocación de marcapaso generador permanente neuroestimulador.

Sólo se pasa de una fase a otra si se objetiva mediante el diario de incontinencia una reducción de ésta en más del $50 \%$

\section{Técnica de implantación}

La técnica de implantación es sencilla y estándar. Hoy en día se prefiere el uso de un electrodo tetrapolar de autoanclaje, implantado con técnica de Seldinger el cual no necesita ser sustituido al colocar el generador definitivo.

En pabellón quirúrgico, con técnica aséptica completa, se coloca al paciente en posición de decúbito prono con elevación de pelvis y flexión parcial 


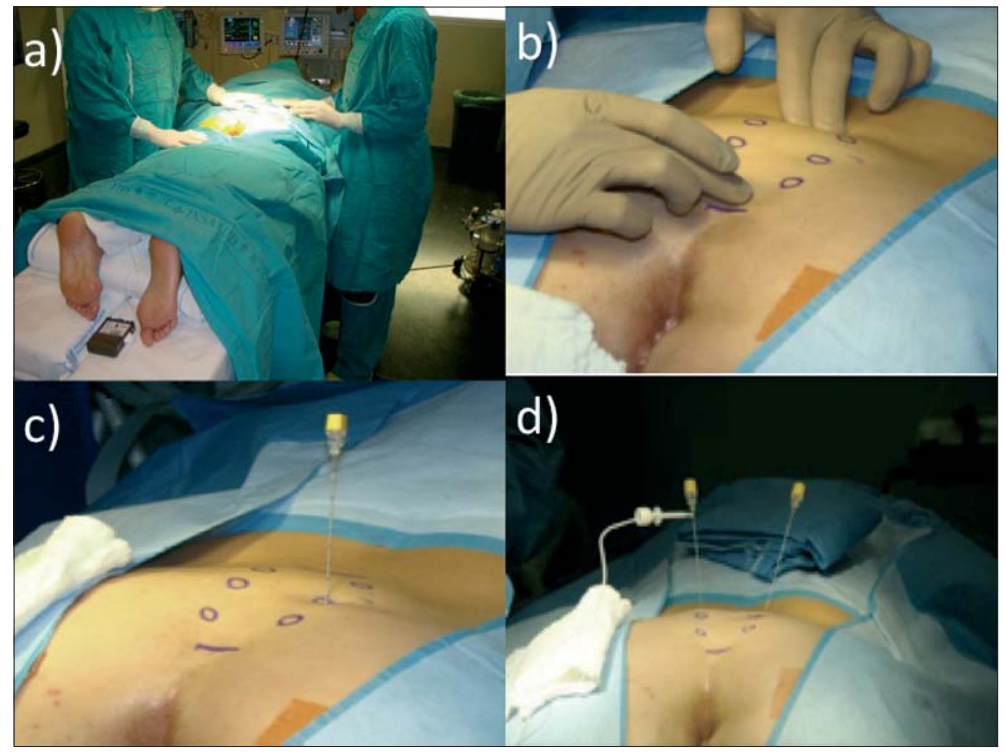

Figura 1. a) posición del paciente; b) identificación de orificios sacros; c) punción $\mathrm{S}_{3}$ izquierda; d) estimulación electrodo $\mathrm{S}_{3}$ derecho.

de extremidades inferiores. Se separan los glúteos con cinta adhesiva y se prepara el campo quirúrgico con técnica estándar, dejando descubierta la zona sacra, ambos glúteos, zona anal y perianal. Asimismo se dejan descubiertos ambos pies para visualizar respuesta motora distal a la prueba de estimulación. Los agujeros sacros se localizan con el paciente en decúbito prono y siguiendo referencias óseas. Se debe contar en pabellón con arco en C para radioscopia intra operatoria (Figuras 1a y 1b).

Bajo anestesia local y sedación superficial se introduce una aguja de estimulación incorporada en el kit de instalación (Medtronic Interstim ${ }^{\circledR}$, Minnesota, USA) en el agujero sacro elegido, con una inclinación de alrededor de 60 grados con respecto al eje horizontal en dirección caudal de manera de que ingrese por uno de los forámenes sacros seleccionados (Figuras 1c y 1d). Para el tratamiento de incontinencia fecal se prefiere estimular $\mathrm{S}_{3}$. Es necesario ubicar adecuadamente la aguja bajo visión directa y radioscópica. Se inicia la estimulación de la raíz sacra seleccionada con un generador externo con estímulos crecientes con una onda de 0,5 Volt $(0,5$ a 10 Volts, frecuencia de $25 \mathrm{~Hz}$ y amplitud de $210 \mu \mathrm{s}$ ) hasta obtener la respuesta sensorial y/o motora esperada, la cual es diferente de acuerdo a la raíz sacra estimulada. Esta respuesta puede implicar contracción del esfínter externo, flexión del antepié, sensación de estimulación vulvar y/o perineal.

Una vez identificado el sitio de estimulación adecuado, se coloca por técnica de Seldinger un electrodo de estimulación tetrapolar (Quadripolar Lead
Kit Medtronic Interstim ${ }^{\circledR}$, Minneapolis, Minnesota, USA), el cual se conecta a una unidad de estimulación externa (Test Stimulator Medtronic Interstim ${ }^{\circledR}$ Minneapolis, Minnesota, USA) (Figura 2).

\section{Protocolo de seguimiento}

El paciente es dado de alta con su estimulador externo funcionando y se le pide que complete un diario de incontinencia. Se controla en forma ambulatoria a los 3,7 y 10 días. Si se aprecia una disminución de los episodios de incontinencia en al menos un 50\% se indica el implante definitivo. Este se coloca, al igual que los generadores marcapasos cardíacos, en posición subcutánea, en la zona

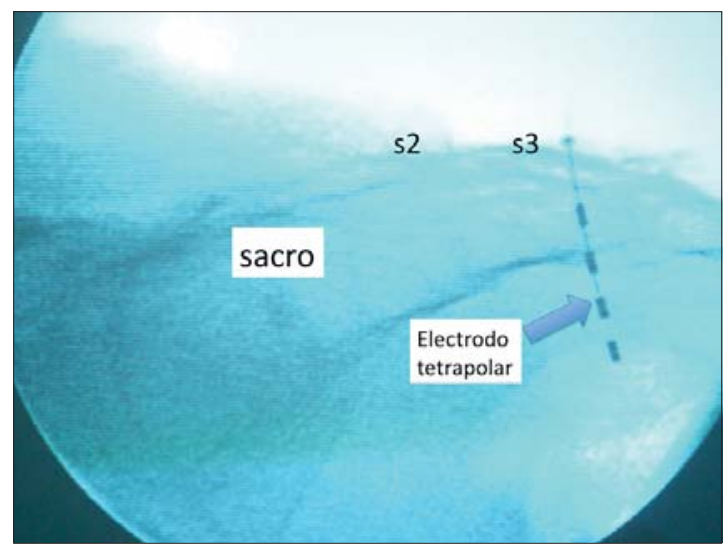

Figura 2. Visión radioscópica de electrodo tetrapolar en $\mathrm{S}_{3}$. 


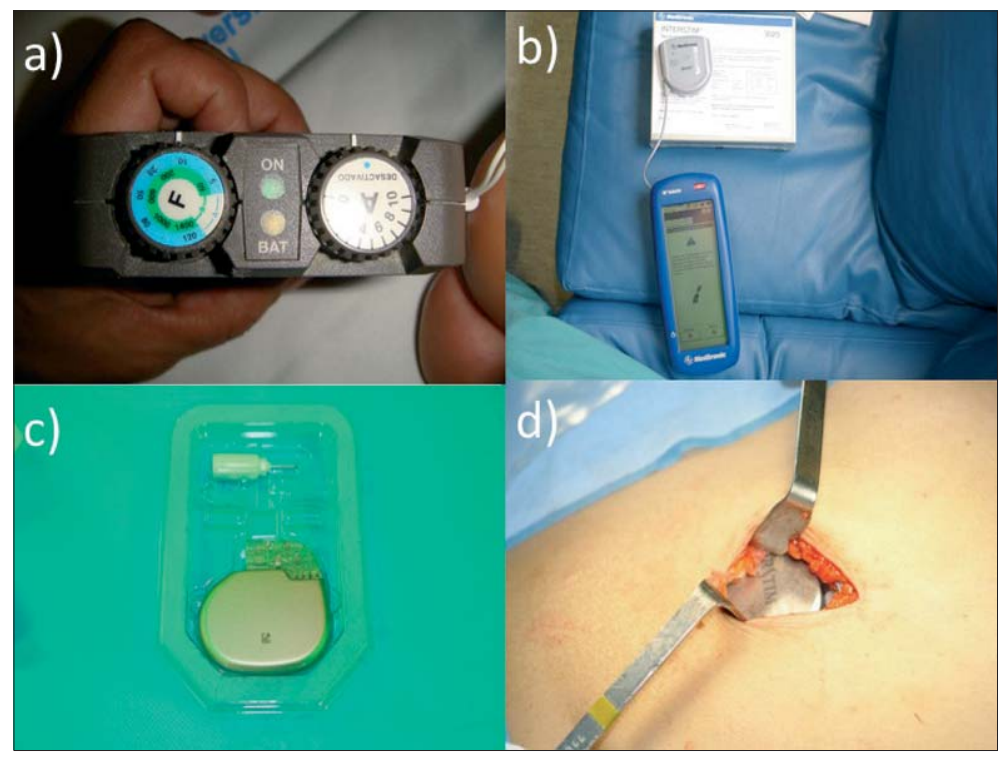

Figura 3. a) Estimulador externo; b) unidad de programación externa; c) marcapaso definitivo; d) marcapaso en bolsillo subcutáneo fosa iliaca derecha.

de fosa iliaca derecha o izquierda según la lateralidad del sitio de estimulación elegido. Este marcapaso es programado en forma percutánea mediante un programador externo, al implantarlo y en los controles ambulatorios (Figura 3).

\section{Discusión}

La continencia anal implica la preservación de un mecanismo complejo que incluye indemnidad anatómica del aparato esfinteriano y elementos de sostén, capacidad de distensión y acomodación rectal adecuada e integridad anatómica y funcional neurológica local y central ${ }^{1,10}$. Ante la presencia de una incontinencia fecal asociada a un defecto esfinteriano, el tratamiento de elección es la esfinteroplastía anal. Sin embargo, en los pacientes en quienes no se encuentra una alteración anatómica del esfínter externo o en quienes la cirugía reparadora ha fallado, la aparición de terapias como la NMS brindan una nueva alternativa terapéutica ${ }^{11}$.

La NMS actúa "modulando" las respuestas motoras, sensitivas y cognitivas asociadas al mecanismo de la defecación. La mayoría de las publicaciones sugieren que la NMS puede mejorar la actividad muscular esfinteriana y conseguir un aumento de presión de reposo. De hecho, los efectos de la estimulación son mucho más complejos que una simple acción sobre las vías motoras eferentes de los esfínteres anales, ya que se plantea que el mecanismo de acción se debería a una modulación de los reflejos sacros, lo que originaría modificaciones en la contractilidad rectal y actuaría también sobre la sensibilidad y distensibilidad del recto ${ }^{10,12}$.

La terapia de NMS fue aprobada por la Food and Drug Administration (EEUU) inicialmente para la incontinencia urinaria en el año 1999 y en 2006 para constipación crónica severa e incontinencia anal. Asimismo la comisión de salud de la Comunidad Europea (EMEA) aprobó su uso y se considera actualmente como terapia innovadora, pero estandarizada no experimental, para el tratamiento de las patologías descritas.

La experiencia a nivel mundial hasta el año 2005 era de más de 3.500 pacientes. Sólo en Europa se han tratado más de 1.700 pacientes en 280 centros.

En su inicio, los criterios de inclusión para la NMS se limitaban a la incontinencia fecal grave con integridad de la conducción nerviosa del pudendo y del esfínter externo. Actualmente se han ampliado sus indicaciones, no estando contraindicada con defectos esfinterianos menores al $50 \%{ }^{13}$.

La evaluación pre operatoria debe incluir al menos:

- Manometría anorrectal.

- Endosonografía anal.

- Diario de incontinencia.

La NMS está contraindicada en:

- Enfermedades de la zona sacra que afecten a la implantación del electrodo como la espina bífida o enfermedad dermatológica.

- Defectos esfinterianos con posibilidad de reparación quirúrgica.

- Grandes defectos que requieren reemplazo esfin- 
teriano con graciloplastía o con el uso de esfínter anal artificial.

- Embarazo.

- Alteraciones de la coagulación.

- Pacientes portadores de marcapaso cardíaco o desfibrilador permanente.

Uno de los aspectos más interesantes de los estudios publicados es que un alto porcentaje (90\%) de los pacientes que se benefician de la NMS temporal van a obtener el mismo beneficio en la fase permanente. Por tanto, la fase de prueba tiene una alta fiabilidad para predecir buenos resultados con la NMS a largo plazo ${ }^{14}$. Por otro lado, un $22 \%$ de los pacientes no responde a esta terapia, lo cual puede ser detectado ya en la fase de prueba.

Existe evidencia en la literatura de pacientes con más de 6 años de seguimiento con buenos resultados mantenidos en el tiempo ${ }^{15}$.

La estimulación sacra no sólo disminuye la frecuencia de los episodios de incontinencia, sino que además consigue que el paciente sea capaz de diferir la evacuación hasta el momento más oportuno, mejorando su calidad de vida.

\section{Evaluación post implante}

El estudio con exámenes de fisiología anorrectal en la fase permanente muestran resultados variables $^{16}$ :

- Aumento de la presión de contracción voluntaria.

- Tendencia a disminuir los umbrales de sensación rectal.

- Disminución clara de la urgencia evacuatoria.

- Cambios en la motilidad rectal y anal medidos por manometría, disminuyendo los complejos de actividad en el recto y el número de relajaciones espontáneas en el esfínter anal interno.

\section{Complicaciones}

Las complicaciones en la fase inicial o temporal son mínimas. Debe considerarse la NMS transitoria como prueba de elección para seleccionar los candidatos a la fase permanente ${ }^{16}$. Las complicaciones más frecuentes son:

- Desplazamiento de los electrodos.

- Infección superficial.

- Ruptura del sistema.

El uso del electrodo de autoanclaje disminuye la posibilidad del desplazamiento y rotura. En la fase permanente, las complicaciones más frecuentes son la dehiscencia e infección de la herida operatoria, el desplazamiento del electrodo y el dolor persistente, que puede obligar al retiro del marcapasos.

\section{Conclusión}

Basados en la revisión de la literatura y con la experiencia de nuestro primer paciente, consideramos que la NMS representa una válida y efectiva alternativa terapéutica en el tratamiento de la incontinencia fecal. Esta técnica permite tratar a pacientes que de otra manera ven comprometida seriamente su calidad de vida.

Además, el hecho de que la NMS es una terapia quirúrgica mínimamente invasiva y que permite la realización de una prueba terapéutica (fase temporal) nos ayuda a seleccionar los pacientes que podrán beneficiarse con este tratamiento.

\section{Referencias}

1. Norton C, Whitehead WE, Bliss DZ, Tries J. Conservative and pharmacological management of faecal incontinence in adults. En: Incontinence. Abrams P., Cardozo L., Khoury S., Wein AJ. Ed. USA, Health Publications: Plymouth 2005; 1521-1563.

2. Zárate A, López-Köstner F, Vergara F, Badilla N, Viviani P. Prevalencia de la incontinencia fecal en centros de salud y casas de reposo. Rev Méd Chile 2008; 136: 867-872.

3. Perry S, Shaw C, McGrother C, Matthews RJ, Assassa RP, Dallosso H, et al. Leicestershire MRC Incontinence Study Team. Prevalence of faecal incontinence in adults aged 40 years or more living in the community. Gut 2002; 50: 480-484.

4. Sultan AH, Kamm MA, Hudson CN, Thomas JM, Bartram CI. Anal-sphincter disruption during vaginal delivery. N Engl J Med 1993; 329: 1905-1911.

5. Bachoo P, Brazelli M, Grant A. Surgery for faecal incontinence in adults. Cochrane Database Syst Rev 2000; (2): CD001757.

6. Devesa JM, Rey A, Hervas PL, Halawa KS, Larrañaga I, Svidler L, et al. Artificial anal sphincter: complications and functional results of a large personal series. Dis Colon Rectum 2002; 45: 1154-1163.

7. Schmidt RA. Application of neurostimulation in urology. Neurourol Urodyn 1988; 7: 585-592.

8. Tanagho EA, Schmidt RA. Bladder pacemaker: Scientific basis and clinical future. Urology 1982; 20: 614-619.

9. Matzel KE, Stadelmaier U, Hohenfellner M, Gall FP. Electrical stimulation for the treatment of faecal incontinence. Lancet 1995; 346: 1124-1127.

10. Muñoz-Duyos A, Montero J, Navarro A, Del Río C, García-Domingo MI, Marco C. Incontinencia fecal: neurofisiología y neuromodulación. Cir Esp 2004; 76: 65-70.

11. Matzel KE, Kamm MA, Stösser M, Baeten CG, Christiansen J, Madoff R, et al. Sacral nerve stimulation 
for fecal incontinence: a multicenter study. Lancet 2004; 363: 1270-1276.

12. Vaizey CJ, Kamm MA, Turner IC, Nicholls RJ, Woloszko J. Effects of short-term sacral nerve stimulation on anal and rectal function in patients with anal incontinence. Gut 1999; 44: 407-412.

13. Leng W, Chancellor M. How Sacral Nerve Stimulation Neuromodulation Works. Urol Clin N Am 2005; 32: 11-18.

14. Dudding TC, Parés D, Vaizey CJ, Kamm MA.
Predictive factors for successful sacral nerve stimulation in the treatment of faecal incontinence: a 10-year cohort analysis. Colorectal Dis 2008; 10: 249-256.

15. Conaghan P, Farouk R. Sacral nerve stimulation can be successful in patients with ultrasound evidence of external anal sphincter disruption. Dis Colon Rectum 2005; 48: 1610-1614.

16. Matzel KE, Stadelmaier U, Hohenberger W. Innovations in fecal incontinence: sacral nerve stimulation. Dis Colon Rectum 2004; 47: 1720-1728. 